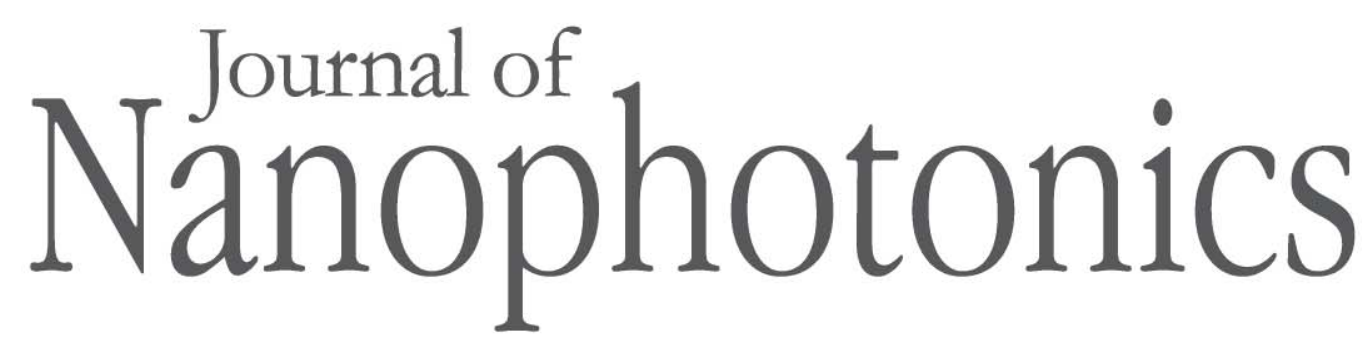

SPIEDigitalLibrary.org/jnp

\title{
Coherent oscillations between orbital angular momentum polariton states in an elliptic resonator
}

Gaël Nardin

Yoan Léger

Barbara Piętka

François Morier-Genoud

Benoît Deveaud-Plédran 


\title{
Coherent oscillations between orbital angular momentum polariton states in an elliptic resonator
}

\author{
Gaël Nardin, Yoan Léger, Barbara Piętka, François Morier-Genoud, \\ and Benoît Deveaud-Plédran \\ Laboratoire d'Optoélectronique Quantique, École Polytechnique Fédérale de Lausanne \\ (EPFL), Station 3, CH-1015 Lausanne, Switzerland \\ gael.nardin@epfl.ch
}

\begin{abstract}
We optically excited the eigenmodes of an elliptic resonator in a semiconductor microcavity. Using a pulsed excitation, we created a superposition of eigenmodes, and imaged the temporal evolution of the coherent emission pattern. A semiconductor quantum well was embedded in the microcavity structure. The system was operated in the strong light matter coupling regime, where the eigenmodes are hybrid half-photonic half-excitonic quasiparticles called exciton polaritons. Oscillations between orbital angular momentum states (or vortex states) were observed, and turned out to be remarkably well described within the Poincaré sphere representation. () 2011 Society of Photo-Optical Instrumentation Engineers (SPIE). [DOI: 10.1117/1.3609825]
\end{abstract}

Keywords: orbital angular momentum; semiconductor microcavity; exciton; polariton; vortex; coherent superposition; ultrafast dynamics; digital holography.

Paper 11053LR received Apr. 1, 2011; revised manuscript received Jun. 15, 2011; accepted for publication Jun. 20, 2011; published online Jul. 21, 2011.

\section{Introduction}

Generation of optical vortices has been demonstrated in different types of lasers like ring resonators, ${ }^{1}$ diode lasers, ${ }^{2}$ and vertical cavity surface emitting lasers (VCSELs). ${ }^{3}$ They have been identified as the transverse modes of cylindrically symmetric optical resonators and often called the $\mathrm{TEM}_{01^{*}}$ "donut" mode, which can be obtained as a superposition of the $\mathrm{TEM}_{10}$ and TEM $\mathrm{M}_{01}$ modes. ${ }^{4-7}$ In semiconductor microcavities, the strong coupling between the optical modes of the planar microcavity and the excitonic resonance of an embedded quantum well (QW) gives rise to hybrid quasiparticles partly made of light, partly made of matter, called exciton polaritons,${ }^{8}$ whose unique properties have triggered interest in fundamental physics, ${ }^{9,10}$ as well as for the implementation of novel all-optical logic gates. ${ }^{11,12}$ The generation of optical vortices strongly coupled to the excitonic resonance (or polariton vortices) has been demonstrated in a patterned microcavity. ${ }^{13,14}$ The vortices, carrying an integer orbital angular momentum state, were the result of a coherent superposition of eigenmodes of an elliptic polariton trap, excited using a continuous wave (cw) laser. In the present work we are considering the case where the confined polariton states are not continuously driven by the laser frequency (like a forced oscillator), but are excited with a laser pulse and let free to evolve. In this situation, when a superposition of states is excited, their phase oscillations at different frequencies will give rise to a time evolution of the emission pattern.

\section{Sample}

The sample under scrutiny is the same as in Refs. $12-14$. It is a patterned GaAs cavity ${ }^{15}$ with one embedded InGaAs QW, sandwiched between two semiconductor distributed bragg reflectors

$1934-2608 / 2011 / \$ 25.00$ @ 2011 SPIE 

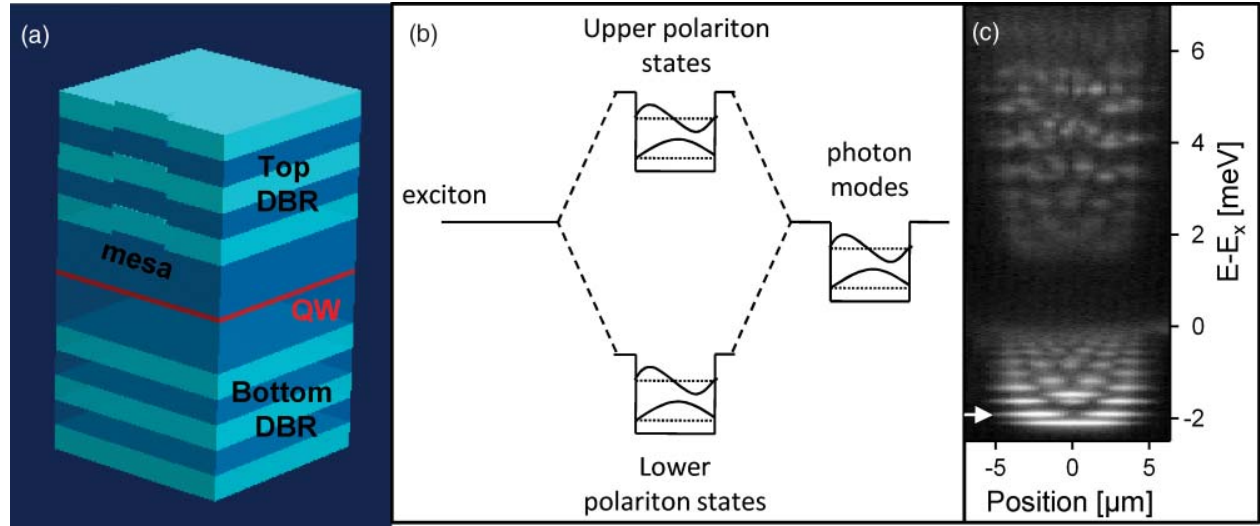

Fig. 1 (a) Scheme of the microcavity sample with mesa (not to scale, only a few DBR pairs are shown). (b) Scheme of the normal mode splitting (or Rabi splitting) arising from the strong coupling between the quantum well excitonic resonance and the transverse photonic modes as a result of the confinement by the mesa. It results in discrete confined transverse modes for the upper and lower polariton branches. (c) Spatially resolved photoluminescence spectrum of the polaritons confined by a mesa of $10 \mu \mathrm{m}$ mean diameter, under nonresonant pumping. Energy is plotted with respect to the bare exciton energy $\left(E_{x}=1.4845 \mathrm{eV}\right)$. Discrete confined states are visible for the lower (below $E_{x}$ ) and upper (above $E_{x}$ ) polariton branches. The white arrow points on the first excited state $(n=1, m=1)$ of the lower polariton branch.

(DBRs). The QW exciton emission wavelength is $835.15 \mathrm{~nm}$, corresponding to an energy of $E_{x}=1.4845 \mathrm{eV}$. The signature of the strong coupling between the exciton and the fundamental cavity mode is a normal mode splitting (or Rabi splitting) of $3.5 \mathrm{meV}$. The polariton traps consist of nearly circular mesas of $6 \mathrm{~nm}$ height that were etched on the microcavity spacer [see Fig. 1(a)], providing a confinement potential of $9 \mathrm{meV}$ for the cavity mode. Discrete confined polariton states are the result of the strong coupling between the optical modes of the nearly cylindrical resonator and the exciton [Fig. 1(b)]. The transverse mode patterns of these zero dimensional (0D) polariton states have been observed in traps of different sizes. ${ }^{16}$ These patterns are similar to the optical mode profiles observed in cylindrical CdTe micropillars. ${ }^{17} \mathrm{~A}$ spatially resolved spectrum of the photoluminescence emitted by the confined states in a $10 \mu$ m mean diameter trap under nonresonant pumping is shown in Fig. 1(c). Discrete eigenstates can be observed for the lower polariton branch (below the bare exciton energy $E_{x}$ ) and the upper polariton branch (above $E_{x}$ ). The linewidth of these states is of the order of $80 \mu \mathrm{eV}$. All further measurements presented in the paper were performed on the first excited state of the lower polariton branch [indicated by a white arrow in Fig. 1(c)], and for a detuning of $\delta \sim 0 \mathrm{meV}$ between the confined photonic mode and the excitonic resonance $E_{x}$.

\section{Poincaré Sphere Representation of an Eigenstate Containing Orbital Angular Momentum}

In circular coordinates, confined states can be described by two numbers $(n, m)$, the well known radial and orbital quantum numbers. In cylindrically symmetric systems, there is a twofold degeneracy between states $\left|m_{+}\right\rangle \propto e^{+i m \phi}$ and $\left|m_{-}\right\rangle \propto e^{-i m \phi}$, which carry an integer orbital angular momentum. Similarly to polarization states, ${ }^{7}$ the coherent superpositions of $\left|m_{+}\right\rangle$and $\left|m_{-}\right\rangle$can be placed on a Poincaré sphere [Fig. 2(a)—for the quantum numbers $(n=1, m=1)$ ]. Equally weighted superpositions give rise to standing wave patterns with $|2 m|$ lobes, whose alignment depends on the phase relation between $\left|m_{+}\right\rangle$and $\left|m_{-}\right\rangle$. For example, the states shown in Fig. 2(a) can be obtained as $|x\rangle=\left|m_{+}\right\rangle+\left|m_{-}\right\rangle,|y\rangle=\left|m_{+}\right\rangle-\left|m_{-}\right\rangle,|d\rangle=\left|m_{+}\right\rangle+i\left|m_{-}\right\rangle$, and $\left|d^{\prime}\right\rangle=\left|m_{+}\right\rangle-i\left|m_{-}\right\rangle$. Reversibly, the $\left|m_{+}\right\rangle$state can be obtained as a superposition of $|x\rangle$ 


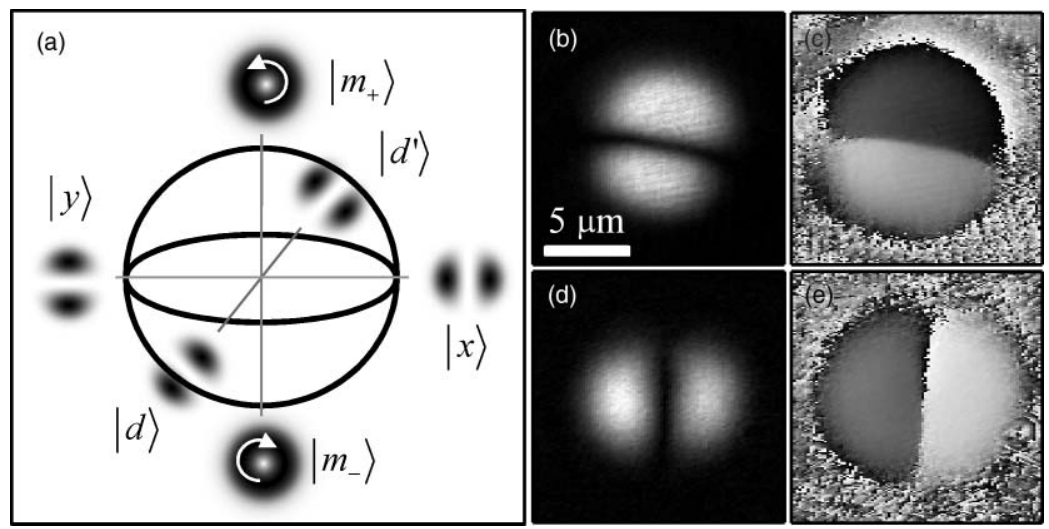

Fig. 2 (a) Poincaré sphere representation of the degenerate $(n=1, m=1)$ state. Coherent superpositions (with equal weights) of the integer orbital angular momentum states $\left|m_{+}\right\rangle$and $\left|m_{-}\right\rangle$yield to a two-lobe pattern, whose orientation depends of the relative phase between $\left|m_{+}\right\rangle$ and $\left|m_{-}\right\rangle$. For this $m=1$ state, the same Poincaré sphere can be used to represent the case of an elliptic trap, for which $|x\rangle$ and $|y\rangle$ are the new eigenstates. (b) Amplitude of the near field emission pattern of $|y\rangle$, measured using the interferometric method described in Sec. 4. (c) Corresponding phase structure, showing a $\pi$-phase shift between the lower and upper lobes. (d) and (e) Same as (b) and (c), but for the $|\boldsymbol{x}\rangle$ state. Amplitude patterns are in a linear gray scale from minimal (black) to maximal (white) intensity. Phase patterns are in a linear gray scale from 0 (black) to $2 \pi$ (white).

and $|y\rangle$, and so on. However, it was shown previously that the polariton traps on the sample under scrutiny are slightly elliptic. ${ }^{15,18}$ This has for effect that the $\left|m_{+}\right\rangle$and $\left|m_{-}\right\rangle$are no longer eigenstates of the trap. The new eigenstates are, for the $(n=1, m=1)$ case, the $|x\rangle$ and $|y\rangle$ states shown in Fig. 2(a). Spatially resolved spectroscopy ${ }^{16,17,19-22}$ allowed to measure an energy splitting between $|x\rangle$ and $|y\rangle$ of $\Delta E=50 \pm 25 \mu \mathrm{eV}$. These eigenstates can be selectively excited using resonant $\mathrm{cw}$ excitation. Their phase and amplitude patterns can be imaged thanks to an interferometric method (detailed in Sec. 4). Near field patterns are shown in Figs. 2(b)-2(e), where the two-lobe structure, as well as the $\pi$-phase between the two opposite lobes, can nicely be observed. Using a cw excitation also allowed to selectively create any kind of superpositions of $|x\rangle$ and $|y\rangle .^{14,18}$

\section{Experimental Setup}

In order to obtain the amplitude and phase structure of the polariton modes shown in Fig. 2, we have used a homodyne imaging setup. ${ }^{13}$ Polariton states were excited from the back side of the sample (held in a cold-finger cryostat at a liquid helium temperature). The coherent emission of the polariton state was collected from the front side of the sample, and interfered on a CD with a reference laser beam. Digital off-axis filtering ${ }^{23}$ allowed then to retrieve the amplitude and phase information of the emission pattern. When using a pulsed excitation instead of a cw one, the situation is very different, as the created coherent superposition is let free to evolve. We use a mode-locked Ti:Sapphire laser, which provides $12 \mathrm{~nm}$ broad, $80 \mathrm{fs}$ long, linearly polarized pulses at a repetition rate of $80 \mathrm{MHz}$. Creating a polariton population with the excitation pulse, we can probe the polariton dynamics by varying the delay $\tau$ between the excitation and reference pulses. Indeed, the reference pulse will interfere on the CCD only with the coherent emission emitted at delay $\tau$. The time resolution of the setup is given by the temporal length of the reference pulse. In order to selectively excite the trapped polariton states, the excitation pulse is tailored to a nearly Gaussian pulse of $140 \mu \mathrm{eV}$ FWHM using a pulse shaper. The corresponding pulse duration is $13 \mathrm{ps}$, which provides the time resolution of the interferometric measurements. 
The interferometric detection is intrinsically linearly polarized due to the linear polarization of the reference laser beam.

\section{Results: Oscillations Between Vortex and Antivortex States}

We use the $140 \mu \mathrm{eV}$ broad pulse obtained from the pulse shaper to excite a superposition of the $|x\rangle$ and $|y\rangle$ eigenstates resulting from the slight ellipticity of the $10 \mu \mathrm{m}$ mean diamater trap. It is very convenient to represent again the superposition in a Poincaré sphere [Fig. 3(a)]. We place the eigenstates $|x\rangle$ and $|y\rangle$ on the vertical axis, and their possible superpositions on the equator of the sphere. As the two eigenstates oscillate with different frequencies, their relative phase will constantly change with time, leading to an evolution of the emission pattern. This evolution can be very easily pictured in the Poincaré sphere representation as a rotation of
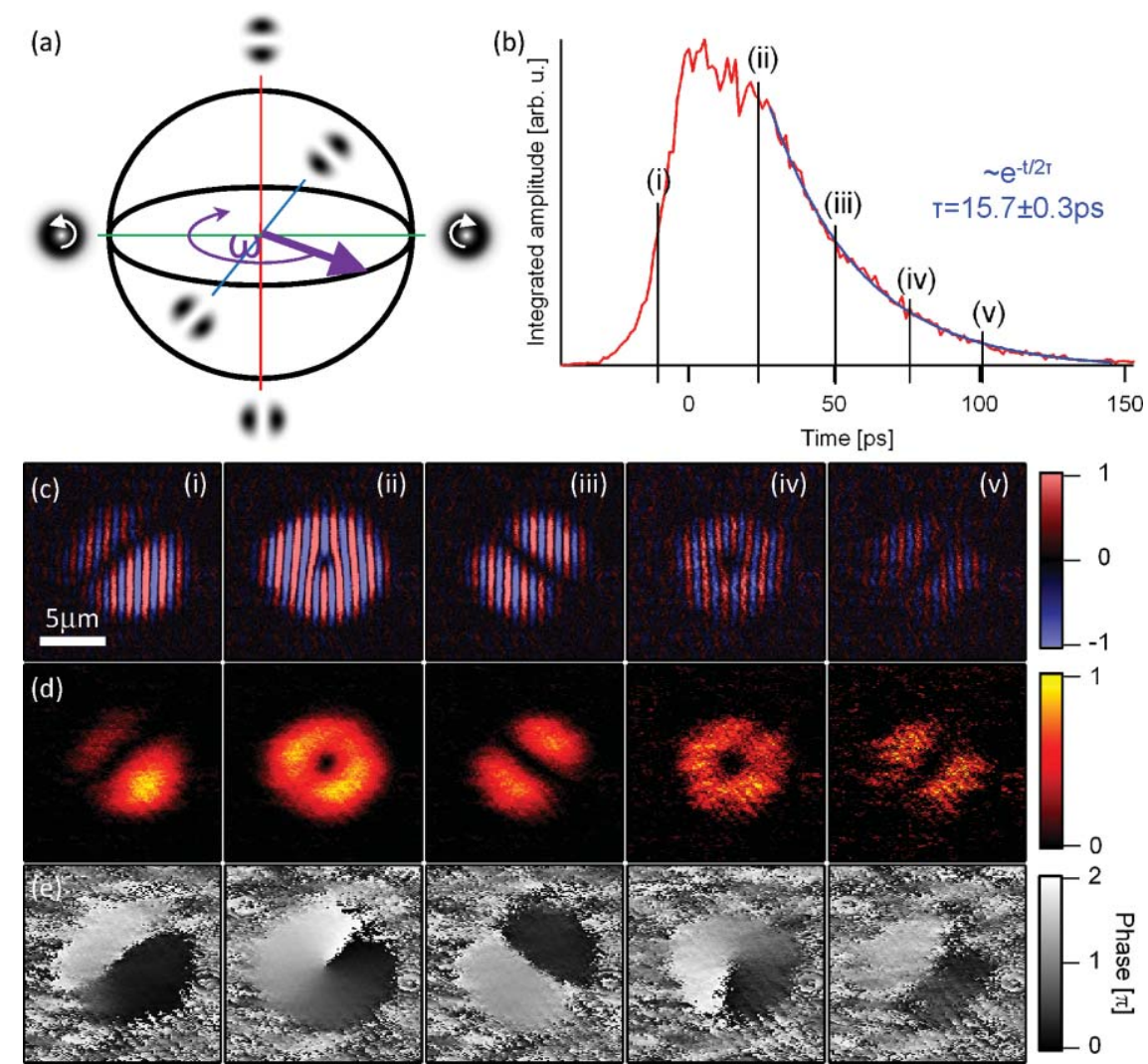

Fig. 3 (a) Poincaré sphere representation of the nearly degenerate $(n=1, m=1)$ state in a nearly circular trap. The eigenstates as a result of the trap ellipticity correspond to the poles of the sphere, and their equally weighted superposition are located on the equator. The time evolution of a superposition can be pictured as a rotation of the state vector around the eigenaxis, at a frequency $\omega=\Delta E / \hbar$, where $\Delta E$ is the energy splitting between the eigenstates $|x\rangle$ and $|y\rangle$. (b) Amplitude $\langle E\rangle$ of the polariton coherent emission field, spatially integrated over the whole polariton emission, versus time. The time zero is given by the estimated zero delay between excitation and reference pulses. In blue: a mono-exponential fit allows to extract a decay time of $\tau=15.7 \pm 0.3 \mathrm{ps}$ for the emission field intensity $\left\langle E^{2}\right\rangle$. The fringes of the recorded interferogram (in a saturated color scale) are displayed in (c), the emission field amplitude in (d), and the emission field phase in (e). The different columns show snapshots of the time evolution of the emission pattern, at times indicated by vertical black lines and a corresponding letter (i)-(v) in (b). This evolution is very well explained by a rotation of the system state vector along the equator of the Poincaré sphere shown in (a). The movie corresponding to (c) is shown in Video 1. 


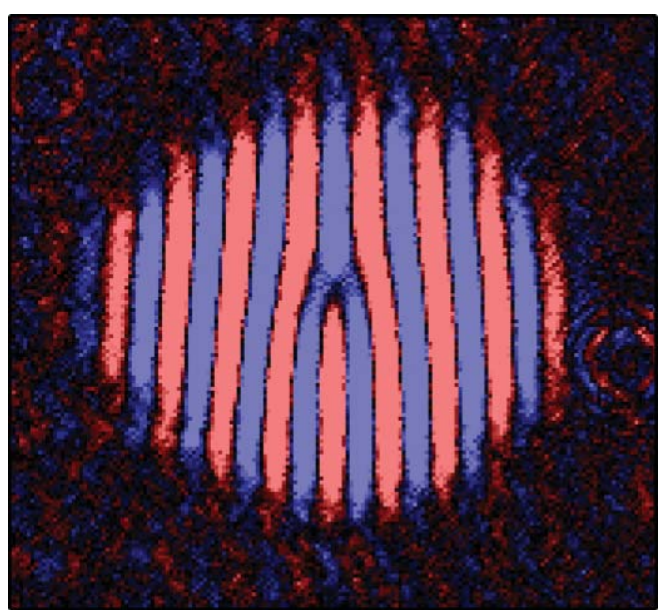

Video. 1: Video corresponding to the snapshots of Fig. 3(c). (MOV, $2.36 \mathrm{MB}$ ) [URL: http://dx.doi.org/10.1117/1.3609825.1].

the state vector of the system around the eigenaxis at a frequency $\omega=\Delta E / \hbar$. This periodical oscillation is analogous to the Rabi oscillations observed when a superposition of upper and lower polaritons is created, or to the spatial oscillations observed between two coupled wells when a superposition of the symmetric and antisymmetric eigenstates is created. It is also analogous to polarization beats that occur in the case of linear polarization splitting, as predicted in subwavelength elliptical cavities, ${ }^{24}$ and observed in the case of linear-transverse (L-T) splitting in planar microcavities. ${ }^{25}$ TE-TM splitting is also expected to periodically generate doubly charged vortices using a spin-to-orbital-angular-momentum conversion mechanism in a planar cavity. ${ }^{26}$ In the case of our $10 \mu \mathrm{m}$ average diameter traps, though, the polarization splitting is too small for polarization beats to be revealed on the time scale of our experiment.

Once a polariton population is injected by the excitation pulse, the time evolution of the coherent emission can be probed by scanning the delay $\tau$ between excitation and reference pulses. We record on a CCD the resulting interference patterns which arise due to a slight tilt of the reference phase front with respect to the image plane. From the recorded interferograms, digital off-axis filtering allows to extract the amplitude and phase of coherent emission pattern emitted at the delay $\tau$. We show in Fig. 2(b) the spatially integrated amplitude $\langle E\rangle$ of the coherent emission with respect to the interferometer delay. It can be seen that after the injection by the $\sim 13$ ps long pulse, the emission intensity $\left\langle E^{2}\right\rangle$ decays with a characteristic decay time of $\tau=15.7 \pm 0.3 \mathrm{ps}$. This time corresponds to the polariton lifetime, which is a combination of the cavity photon escape rate and the exciton lifetime. Images of the coherent emission amplitude, at the delays indicated by vertical black lines and letters (i)-(v) in Fig. 3(b), are shown in Figs. 3(c)-3(e). The fringes of the interferogram (in a saturated color scale) are displayed in Fig. 3(c), the emission field amplitude in Fig. 3(d), and the emission field phase in Fig. 3(e).

In the first column (i), the polariton injection is shown. It displays a diagonal state $|d\rangle$, indicating that a well balanced (i.e., with similar weights) superposition of $|x\rangle$ and $|y\rangle$ is created. Columns (ii) to (v) show the free evolution of the polariton field emission pattern, with an interval between each picture of $\sim 25 \mathrm{ps}$. A decrease of the signal intensity is observed with time, due to the finite polariton lifetime measured in Fig. 3(b). In the second column (ii), the polariton emission pattern shows an integer orbital angular momentum state (or vortex state) $\left|m_{+}\right\rangle$, characterized by a donut shape. The interferogram displays a fork-like dislocation, indicating the presence of a phase singularity. A $2 \pi$-phase shift is visible in the phase structure when circumventing the donut core. The third column (iii) shows a $\left|d^{\prime}\right\rangle$ state [orthogonally aligned with respect to the $|d\rangle$ state of (i)], featuring a $\pi$-phase difference between its two lobes. An anti-vortex state $\left|m_{-}\right\rangle$is visible in (iv), and a diagonal state $|d\rangle$ again in (v). The system 
state vector has therefore made a full rotation along the Poincare sphere equator. The rotation period is $T \sim 4 \times 25 \mathrm{ps}=100 \mathrm{ps}$ (with a $\sim 13 \mathrm{ps}$ resolution), corresponding to an oscillation frequency of $\sim 10 \mathrm{GHz}$. It allows to estimate for this trap the energy splitting between $|x\rangle$ and $|y\rangle: \Delta E=\hbar \omega=\hbar 2 \pi / 100=41 \pm 5 \mu \mathrm{eV}$, in agreement with the $50 \pm 25 \mu \mathrm{eV}$ generally measured using spatially resolved spectroscopy.

\section{Conclusion}

In conclusion, we have shown in this work the imaging of the time evolution of a coherent superposition of light-matter polariton states in a semiconductor microcavity. In particular, a periodical oscillation between vortex and anti-vortex states has been evidenced in the elliptic polariton trap. The observed phenomenon is remarkably well described by a rotation of the system state vector around the eigenaxis in the Poincare sphere representation of an integer orbital angular momentum state. The measured oscillation frequency of $\sim 10 \mathrm{GHz}$ corresponds to the energy splitting between the eigenstates of the elliptic trap. We would like to underline that although the semiconductor microcavity was operated in the strong coupling regime, the reported phenomenology was observed under low excitation density, and can be thus described in terms of linear optics. However, although not addressed in the present manuscript, nonlinearities originating from the strong coupling with the QW exciton have been reported on the same sample, and used to realize multivalued spin switching. ${ }^{12}$ These nonlinearities have also been shown to modify the spatial mode profiles in confined polariton condensates. ${ }^{22}$

\section{Acknowledgments}

We thank O. El Daïf for the design and fabrication of the sample. We acknowledge support by the Swiss National Research Foundation through the "NCCR Quantum Photonics."

\section{References}

1. M. Brambilla, F. Battipede, L. A. Lugiato, V. Penna, F. Prati, C. Tamm, and C. O. Weiss, "Transverse laser patterns. I. Phase singularity crystals," Phys. Rev. A 43, 5090-5113 (1991).

2. Y. F. Chen and Y. P. Lan, "Transverse pattern formation of optical vortices in a microchip laser with a large fresnel number," Phys. Rev. A 65, 013802 (2001).

3. F. B. De Colstoun, G. Khitrova, A. V. Fedorov, T. R. Nelson, C. Lowry, T. M. Brennan, B. G. Hammons, and P. D. Maker, "Transverse modes, vortices and vertical-cavity surfaceemitting lasers," Chaos, Solitons and Fractals 4, 1575-1596 (1994).

4. J. E. Epler, S. Gehrsitz, K. H. Gulden, M. Moser, H. C. Sigg, and H. W. Lehmann, "Mode behavior and high resolution spectra of circularly-symmetric GaAs-AlGaAs air-post vertical cavity surface emitting lasers," Appl. Phys. Lett. 69, 722-724 (1996).

5. C. Degen, W. Elsässer, and I. Fischer, "Transverse modes in oxide confined VCSELs: Influence of pump profile, spatial hole burning, and thermal effects," Opt. Express 5, 38-47 (1999).

6. M. Padgett, J. Arlt, N. Simpson, and L. Allen, "An experiment to observe the intensity and phase structure of Laguerre-Gaussian laser modes," Am. J. Phys. 64, 77-82 (1996).

7. M. J. Padgett and J. Courtial, "Poincaré-sphere equivalent for light beams containing orbital angular momentum," Opt. Lett. 24, 430-432 (1999).

8. C. Weisbuch, M. Nishioka, A. Ishikawa, and Y. Arakawa, "Observation of the coupled exciton-photon mode splitting in a semiconductor quantum microcavity," Phys. Rev. Lett. 69, 3314-3317 (1992).

9. A. Amo, J. Lefrère, S. Pigeon, C. Adrados, C. Ciuti, I. Carusotto, R. Houdré, E. Giacobino, and A. Bramati, "Superfluidity of polaritons in semiconductor microcavities," Nat. Phys. 5, 805-810 (2009). 
10. K. G. Lagoudakis, T. Ostatnicky, A. V. Kavokin, Y. G. Rubo, R. Andre, and B. DeveaudPlédran, "Observation of half-quantum vortices in an exciton-polariton condensate," Science 326, 974-976 (2009).

11. T. C. H. Liew, A. V. Kavokin, and I. A. Shelykh, "Optical circuits based on polariton neurons in semiconductor microcavities," Phys. Rev. Lett. 101, 016402 (2008).

12. T. K. Paraïso, M. Wouters, Y. Léger, F. Morier-Genoud, and B. Deveaud-Plédran, "Multistability of a coherent spin ensemble in a semiconductor microcavity," Nat. Mater 9, 655-660 (2010).

13. G. Nardin, Y. Léger, B. Pietka, F. Morier-Genoud, and B. Deveaud-Plédran, "Phaseresolved imaging of confined exciton-polariton wave functions in elliptical traps," Phys. Rev. B 82, 045304 (2010).

14. G. Nardin, K. G. Lagoudakis, B. Pietka, F. Morier-Genoud, Y. Léger, and B. DeveaudPlédran, "Selective photoexcitation of confined exciton-polariton vortices," Phys. Rev. B 82, 073303 (2010).

15. O. El Daï, A. Baas, T. Guillet, J.-P. Brantut, R. I. Kaitouni, J. L. Staehli, F. Morier-Genoud, and B. Deveaud, "Polariton quantum boxes in semiconductor microcavities," Appl. Phys. Lett. 88, 061105 (2006).

16. G. Nardin, R. Cerna, T. K. Paraïso, B. Pietka, Y. Léger, O. E. Daif, F. Morier-Genoud, and B. Deveaud-Plédran, "Probability density tomography of microcavity polaritons confined in cylindrical traps of various sizes," Superlattices Microstruct. 47, 207-212 (2010).

17. K. Sebald, C. Kruse, and J. Wiersig, "Properties and prospects of blue-green emitting II-VI-based monolithic microcavities," Phys. Status Solidi B 246, 255-271 (2009).

18. R. Cerna, D. Sarchi, T. K. Paraïso, G. Nardin, Y. Léger, M. Richard, B. Pietka, O. El Daif, F. Morier-Genoud, V. Savona, M. T. Portella-Oberli, and B. Deveaud-Plédran, "Coherent optical control of the wave function of zero-dimensional exciton polaritons," Phys. Rev. B 80, 121309 (2009).

19. G. Nardin, T. K. Paraïso, R. Cerna, B. Pietka, Y. Léger, O. E. Daif, F. Morier-Genoud, and B. Deveaud-Plédran, "Probability density optical tomography of confined quasiparticles in a semiconductor microcavity," Appl. Phys. Lett. 94, 181103 (2009).

20. D. N. Krizhanovskii, K. G. Lagoudakis, M. Wouters, B. Pietka, R. A. Bradley, K. Guda, D. M. Whittaker, M. S. Skolnick, B. Deveaud-Plédran, M. Richard, R. André, and L. S. Dang, "Coexisting nonequilibrium condensates with long-range spatial coherence in semiconductor microcavities," Phys. Rev. B 80, 045317 (2009).

21. D. Sanvitto, A. Amo, L. Viña, R. André, D. Solnyshkov, and G. Malpuech, "Excitonpolariton condensation in a natural two-dimensional trap," Phys. Rev. B 80, 045301 (2009).

22. L. Ferrier, E. Wertz, R. Johne, D. D. Solnyshkov, P. Senellart, I. Sagnes, A. Lemaître, G. Malpuech, and J. Bloch, "Interactions in confined polariton condensates," Phys. Rev. Lett. 106, 126401 (2011).

23. T. Kreis, Handbook of Holographic Interferometry: Optical and Digital Methods, WileyVCH, Weinheim (2004).

24. I. Iorsh, A. Koreshkova, N. Kaliteevskaya, A. Kavokin, R. Abram, S. Brand, and M. Kaliteevski, "Polarization beats in a pillar microcavity," Superlattices Microstruct. 47, 24-28 (2010).

25. W. Langbein, I. Shelykh, D. Solnyshkov, G. Malpuech, Y. Rubo, and A. Kavokin, "Polarization beats in ballistic propagation of exciton-polaritons in microcavities," Phys. Rev. B 75, 075323 (2007).

26. T. C. H. Liew, A. V. Kavokin, and I. A. Shelykh, "Excitation of vortices in semiconductor microcavities," Phys. Rev. B 75, 241301 (2007).

Biographies and photographs of the authors not available. 